Rev Cuid. 2020; 11(1): e798

\title{
Representaciones sociales del cuidar mediante la prescripción enfermera en la red de atención primaria de salud
}

\author{
Social representations of nursing through nurse prescription in the primary healthcare \\ network
}

\section{Representações sociais do cuidado mediante a prescrição enfermeira na rede de atendimento primário em saúde}

\author{
Roberto Álvarez San Martín', Edgardo Renán Álvarez Muñoz ${ }^{2}$, \\ Fernando Sebastián Malhue Torres ${ }^{3}$, Camilo Rodolfo Moncada Rivera ${ }^{4}$
}

Histórico

Recibido:

1 de marzo de 2019

Aceptado:

2 de octubre de 2019

1 Universidad Santo Tomás, Escuela de Enfermería, Facultad de Salud. Teтuco, Chile. Autor de Correspondencia. E-mail: robertoalvarez@santotomas.cl.

https://orcid.org/0000-0003-0402$482 X$

2 Universidad Santo Tomás, Escuela de Enfermería, Facultad de Salud. Teтисо, Chile. E-mail: edgardr07@gmail.com

https://orcid.org/0000-0002-28827494

3 Universidad Santo Tomás, Escuela de Enfermería, Facultad de Salud. Teтисо, Chile. E-mail: f.malhuetorres@hotmail.com https://orcid.org/0000-0002-02201856

4 Universidad Santo Tomás, Escuela de Enfermería, Facultad de Salud. Temuco, Chile. E-mail: mylomoncada@gmail.com https://orcid.org/0000-0002-8007703 Introducción: Frente a una moción parlamentaria que pretende modificar el código sanitario para permitir a los enfermeros tener la facultad legal de prescribir fármacos y dispositivos sanitarios surge la inquietud de analizar la representación social que los enfermeros de atención primaria de salud otorgan a la temática. Objetivo: Describir los componentes figurativos (campo de representación), simbólico (información) y afectivo (actitudinal) del cuidar mediante la prescripción enfermera, que tienen los profesionales de enfermería de la red de atención primaria de salud. Materiales y Métodos: Investigación descriptiva con enfoque cualitativo y diseño de teoría fundamentada, realizada en ocho enfermeras de la red de atención primaria de salud a través de entrevista semiestructurada. Resultados: En la representación social de la prescripción enfermera surgen cinco categorías: gestionar el cuidado, beneficios sociales de la prescripción, restricción en la prescripción, formación profesional del enfermero en prescripción y valor atribuido a la prescripción. Discusión: Las enfermeras de atención primaria, entienden el fenómeno de la prescripción como una indicación de enfermería que se encuentra influenciada por factores asociados a la formación farmacológica de pregrado y la experiencia laboral, similar a lo reportado en algunos estudios en Iberoamérica. Conclusiones: Las enfermeras consideran que la prescripción es un elemento positivo para la entrega de cuidados y que contribuye a la resolutividad en la atención de salud, sin embargo, para llevarla a cabo se requiere de una formación adecuada y de un respaldo legal claro.

Palabras clave: Atención Primaria de Salud; Chile; Enfermeros; Prescripciones de Medicamentos.

Abstract

Introduction: Considering that a parliamentary motion seeks to modify the health code to allow nurses to have the legal power to prescribe drugs and health devices, it is necessary to analyze the social representation that primary healthcare nurses give to prescription. Objective: To describe the figurative (representation field), symbolic (information) and affective (attitudinal) nursing components by means of nurse prescription used by primary health care nursing professionals. Materials and Methods: A descriptive qualitative research with a grounded theory research design was conducted with eight nurses from the primary healthcare network through a semi-structured interview. Results: Five categories were created based on the social representation of nurse prescription: care management, social benefits of prescription, prescription restriction, professional nurse training in prescription, and value attributed to the prescription. Discussion: Primary care nurses perceive the prescription phenomenon as a nursing indication that is influenced by factors associated with undergraduate pharmacology training and work experience, similar to that reported in some studies in Ibero-America. Conclusions: Nurses consider that nurse prescription is a positive element for care delivery, contributing to the resolutive capacity of healthcare. However, adequate training and clear legal support are required to do so. Key words: Primary Health Care; Chile; Nurses, Male; Drugs Prescriptions.

Introdução: Frente a uma moção parlamentar que objetiva emendar o código sanitário para permitir que os enfermeiros tenham a faculdade legal de prescrever fármacos e dispositivos sanitários surge a inquietude de analisar a opinião dos enfermeiros de atendimento primário em saúde sobre a sua representação social a respeito dessa questão. Objetivo: Descrever os componentes figurativo (campo de representação), simbólico (informação) e afetivo (atitudinal) do cuidar mediante a prescrição de enfermagem que os profissionais da rede de enfermagem detêm na rede de atendimento primário em saúde. Materiais e Métodos: Pesquisa descritiva com abordagem qualitativa e desenho de teoria fundamentada, realizada com oito enfermeiras da rede de atendimento primário em saúde através de uma entrevista semiestruturada. Resultados: Na representação social da prescrição de enfermagem surgem cinco categorias: administrar o cuidado, os benefícios sociais da prescrição, restrições na prescrição, formação profissional em prescrição do enfermeiro e valor atribuído à prescrição. Discussão: As enfermeiras de atendimento primário compreendem o fenómeno da prescrição como uma indicação de enfermagem influencia por fatores associados à formação farmacológica de pré-graduação e a experiência de trabalho, semelhante àquela relatada em vários estudos na Ibero-América. Conclusões: As enfermeiras consideram que a prescrição é um elemento positivo para a entrega de cuidados e que contribui com a resolutividade no atendimento em saúde, no entanto, para cumprir essa tarefa é preciso contar com uma formação adequada e um fundamento legal claro. Palavras chave: Atenção Primária à Saúde; Chile; Enfermeiros; Prescrições de Medicamentos.

Como citar este artículo: Álvarez RSM, Álvarez ERM, Malhue FST, Moncada CRR. Representaciones sociales del cuidar mediante la prescripción enfermera en la red de atención primaria de salud. Rev Cuid. 2020; 11(1): e798. http://dx.doi.org/10.15649/cuidarte.798

(c) (1) () (C)2020 Universidad de Santander. Este es un artículo de acceso abierto, distribuido bajo los términos de la licencia Creative Commons Attribution (CC BY-NC 4.0), que permite el uso ilimitado, distribución y reproducción en cualquier medio, siempre que el autor original y la fuente sean debidamente citados. 


\section{INTRODUCCIÓN}

Una de las actividades con la que se identifica socialmente al profesional de enfermería es la administración de medicamentos o fármacos. En este contexto es donde surge la prescripción enfermera como parte del desarrollo disciplinar ${ }^{1}$. Según Gómez, Alfonso, Hernández y Gómez ella "es el acto de decisión en cualquier aspecto relacionado con un tratamiento específico, incluida la farmacología y la posología”ㄹ. En la actualidad los enfermeros pueden hacer uso legal de la prescripción de medicación en Canadá, Australia, Finlandia, Irlanda, Nueva Zelanda, Noruega, Sudáfrica, los Países Bajos, Suecia, Reino Unido y Estados Unidos ${ }^{3}$. Asumir la prescripción ha sido favorable tanto para el enfermero - al otorgarle más autonomía profesional ${ }^{4,5}$ y una mejor comunicación profesional ${ }^{\underline{6}}$-, como para los pacientes, quienes refieren mayor o igual grado de satisfacción ${ }^{\underline{T}}$ con la atención brindada por enfermeros en comparación con la atención proporcionada por médicos ${ }^{3}$, y a los médicos les ha permitido reducir la carga laboral.

Por otra parte, la evidencia indica que la insuficiente formación de pregrado en el área farmacológica $^{-}$ha obligado a aumentar la oferta de formación y/o especialización de postgrado para que los enfermeros puedan prescribir ${ }^{9}$ Así, en Australia se debe cursar un magíster en el área, en Nueva Zelanda hay que contar con 3 años de experiencia y en Reino Unido hay que realizar una pasantía de 26 días teóricos y una práctica de 12 días
Para el caso chileno, Contreras y Martínez ${ }^{10}$ plantean que el contexto de envejecimiento poblacional, la mayor esperanza de vida y la creciente incidencia de enfermedades crónicas no transmisibles han conducido a que la enfermera de atención primaria sea responsable de la educación sobre los efectos adversos o colaterales de los fármacos y su adecuada prescripción. Sin embargo, en Chile el profesional enfermero no está facultado legalmente para la prescripción de fármacos en ninguno de los niveles de atención. Ante esto, existe una moción parlamentaria para modificar el código sanitario e incorporar la prescripción de medicamentos, cuidados, productos sanitarios e insumos como una parte esencial de los servicios profesionales de la enfermera ${ }^{11}$.

Al respecto, algunos estudios evidencian que la prescripción enfermera de fármacos es una práctica necesaria para la atención primaria de salud (APS), desde que el profesional de la salud trata con la enfermedad y el paciente ${ }^{12}$. Ante esta situación se hace necesario conocer el pensamiento social que tienen las enfermeras frente a la prescripción de fármacos.

Arribar al conocimiento del pensamiento social puede ser alcanzado de diversas formas. Una de ellas es mediante el estudio de las representaciones sociales, opción asumida en esta investigación. Por definición, las representaciones sociales mediante la elaboración de comportamientos y comunicación intersubjetiva ${ }^{13}, \underline{14}$, permiten anticipar la forma de actuar de un grupo en relación con un fenómeno $\frac{15}{}$. Así, la importancia de esta investigación es que aporta antecedentes empíricos orientados a predecir el comportamiento 
que tendrán las enfermeras de atención primaria al otorgárseles la facultad legal de prescribir fármacos.

La pregunta de investigación que orientó el presente estudio fue: ¿Cuál es la representación social frente a la prescripción enfermera de los profesionales de enfermería de la red de atención primaria de salud? Para dar respuesta a este cuestionamiento, el objetivo del estudio fue describir los componentes figurativo (campo de representación), simbólico (información) y afectivo (actitudinal) del cuidar mediante la prescripción enfermera, que tienen los profesionales de enfermería de la red de atención primaria de salud en la comuna de Temuco (La Araucanía, Chile).

\section{MATERIALES Y MÉTODOS}

En relación con el dominio sobre equipo de investigación y reflexividad, la totalidad del equipo es de género masculino. Sobre la relación del equipo de investigación con las participantes, se estableció una relación previa al inicio de la recogida de datos con el único fin de informar sobre el estudio, su enfoque y objetivos, así como de las razones que lo motivaron y las credenciales académicas de los investigadores.

En cuanto al diseño del estudio, se trata de una investigación cualitativa, de corte interpretativo y alcance descriptivo. Se siguió un diseño de teoría fundamentada ${ }^{16,17}$. Este diseño fue sometido a evaluación de Comité de Ética Científica de la institución de educación superior, bajo el código CRTT N9 R. ALVAREZ-ENF-2018.
Sobre la selección de participantes, la población de la investigación corresponde a enfermeras que se desempeñan en la red de atención primaria de salud en la comuna de Temuco (Chile). La muestra se conformó de enfermeras que cumplieran con los siguientes criterios de inclusión: contar con título profesional otorgado por una universidad nacional o extranjera reconocida por el Ministerio de Educación de Chile, desempeñarse en la red de atención primaria de salud de Temuco, realizar funciones en el área asistencial y haber aceptado voluntariamente participar en la investigación; se excluyó a aquellas que, cumpliendo con los criterios de inclusión, contaran con un contrato de jornada laboral menor a 22 horas semanales, tuvieran menos de 2 años de experiencia en atención primaria o desempeñen labores de docencia en la universidad desde la cual se realizó la investigación (excepto tutores de internado). El contacto se realizó mediante un informante clave y la estrategia de selección fue de casos múltiples, siendo abordados desde un enfoque cara a cara. El tamaño de la muestra estuvo sujeto al nivel de saturación teórica, el cual se alcanzó con 8 participantes. Cada una de las participantes en el estudio firmó dos copias de documento de consentimiento informado, cuyo modelo fue proporcionado por el Comité de Ética Científica institucional, del cual una copia quedó en poder de la participante y una segunda copia en poder del investigador responsable (principal). No hubo potenciales participantes que al ser contactados se hayan negado a ser parte del estudio.

La recolección de la información se realizó en dependencias de los centros de salud donde tra- 
bajan las participantes, en días y horarios convenidos anticipadamente y en un horario que no interfiera o afectara en su jornada laboral; durante las entrevistas no hubo presencia de personas diferentes a los investigadores y participantes. Según género, la totalidad de la muestra estuvo conformada por mujeres.

Con respecto a la recopilación de datos, se utilizó una entrevista semiestructurada que se enfocó en los tres componentes de una representación social (figurativo, simbólico y afectivo) del cuidar mediante la prescripción enfermera. La entrevista contó con una guía de preguntas, la cual fue sometida a un proceso de validación por dos jueces externos con experiencia en investigación cualitativa.

Las entrevistas se realizaron en una única sesión, de una extensión aproximada de 40 minutos, cada una de las cuales fue grabada en registro de audio, para su posterior transcripción digital a fin de continuar con el proceso de análisis. No fue necesario repetir alguna entrevista. Después de cada entrevista, cada investigador tomó notas de campo a fin de contar con información complementaria para la fase de análisis, así como de control para efectos de la discusión en torno a la saturación de los datos. Si bien, las transcripciones no fueron devueltas a las participantes, sí se les hizo entrega de los resultados finales de la investigación vía correo electrónico.

Para el proceso de análisis y hallazgos se utilizó la técnica de diseño sistemático $\frac{18}{}$, recurriendo a codificación abierta por comparación constante, codificación axial y codificación selectiva. Al tér- mino de la primera fase del análisis los investigadores proporcionaron un árbol de codificación y categorías de análisis. Estas categorías emergieron del análisis mismo, teniendo como referencia para su organización los objetivos específicos del estudio y que corresponden a los componentes teóricos de una representación social. Posterior a la fase de análisis se utilizó Cmap Tools, software para la creación y gestión de mapas conceptuales.

Para la elaboración del informe final del estudio, en sintonía con el diseño de teoría fundamentada, se recurrió al uso códigos vivos, y a las participantes se identifica con un código alfanumérico que asegura su anonimato y la confidencialidad de datos personales. Asimismo, la información presentada resguarda la consistencia de los datos y los hallazgos, y se expresan en forma clara y precisa los temas principales, así como también se hace referencia a temáticas menores surgidas en el transcurso de la investigación.

En cuanto a criterios de rigor científico, se recurrió a los de dependencia, credibilidad, transferencia y confirmabilidad ${ }^{19}$. Se realizó también triangulación de investigadores y fuentes.

Con respecto a las consideraciones éticas, se adoptaron los criterios de Ezekiel Emanuel para la ética en investigación ${ }^{20}$ : valor, validez científica, selección equitativa de los sujetos, proporción favorable riesgo-beneficio, evaluación independiente, consentimiento informado y respeto a los sujetos participantes. Complementariamente, durante el desarrollo del estudio se tuvieron en consideración las pautas éticas internacionales para la investigación biomédica en seres humanos del 
Consejo de Organizaciones Internacionales de las Ciencias Médicas (COICS) y la Organización Mundial de la Salud (OMS) ${ }^{21}$, así como los principios éticos para las investigaciones médicas en seres humanos de la Declaración de Helsinki de la Asamblea Médica Mundial22, y los principios éticos de respeto a las personas del Informe Belmont para la protección de los sujetos humanos de investigación $\underline{23}$.

\section{RESULTADOS}

Este estudio se centró en la descripción de los componentes figurativo, simbólico y afectivo del cuidar mediante la prescripción enfermera.
En la primera dimensión, figurativa o campo de representación, las enfermeras identifican tres categorías de análisis: gestionar el cuidado, beneficios sociales de la prescripción y restricción en la prescripción.

La categoría gestionar el cuidado se estableció con respecto a los pensamientos colectivos en torno a la imagen del concepto de prescripción enfermera, la cual es entendida como una herramienta para entregar los cuidados de enfermería. De ella se desprende la subcategoría indicación. Ésta, fue elaborada como el elemento mediante el cual las enfermeras de atención primaria pueden hacer entrega de cuidados a través de una acción tanto farmacológica como no farmacológica.

“... yo me imagino que la prescripción enfermera es todo lo que tiene que ver con indicaciones desde el punto de la enfermería, todo lo que tenga que ver con gestión del cuidado, pero con enfoque de enfermería y si hablan de prescripción a mí me surge la idea de que también tiene que ver con los medicamentos" (E4).

"Es la indicación de acuerdo a lo que yo vea según las necesidades y según las capacidades que yo tenga respecto a la indicación y conociendo los efectos del medicamento que pueda indicar, por eso tiene que estar acotado” (E6).

"Es la acción de poder dar la indicación de un medicamento o de un dispositivo porque no está tan solo centrado en la parte fármaco, sino en un dispositivo de uso clínico que sea necesario para la atención del usuario” (E7).

La categoría beneficios sociales de la prescripción se estableció en relación con cómo las enfermeras de atención primaria visualizan la prescripción enfermera como una herramienta que tiene repercusión a nivel social. A partir de ella emerge la subcategoría resolutividad. Ésta fue construida con base en la visión que las enfermeras comparten sobre cómo la prescripción llega- ría a transformarse en una herramienta, mediante la cual el paciente es beneficiado por una enfermera que posee una facultad que permite evitar su derivación a un médico; desde la perspectiva de las enfermeras, esto se traduce en ahorro de tiempo, descongestión del sistema de atención y aumento de las horas disponibles del médico. 
"Desde el bienestar social... de disminuir los plazos... de ese aspecto como social, yo creo que aportaría, y obviamente es como una cascada, una cosa lleva a la otra ... porque se podría derivar como los casos más simples del área de enfermería, se podría dar más horas de médico, de morbilidad, así vamos sacando y una cosa, como que lleva a la otra" (E1).

"Es una ganancia porque por lo que el usuario [paciente] va a tener el acceso a través de un profesional que va a poder satisfacer una necesidad en un momento determinado sin que tenga que intervenir una segunda persona o sin que él tenga que recurrir a una oportunidad siguiente" (E7).

La categoría restricción en la prescripción se estableció en relación con la forma en que las enfermeras de atención primaria visualizan a la prescripción enfermera en cuanto a la regulación legal de la acción profesional. De ella surgen las subcategorías limitaciones en la prescripción y aplicación en área de desempeño.
La subcategoría limitaciones en la prescripción hace referencia a cómo las enfermeras establecen que la prescripción farmacológica en enfermería debe estar acotada a un grupo específico de fármacos, eliminando la facultad de total libertad en la prescripción, la cual se encuentra directamente vinculada con la subcategoría responsabilidad del componente afectivo del cuidar a través de la prescripción enfermera.

“... pero de acuerdo a la actividad que uno hace, si uno debiese tener más libertad de prescribir, pero de manera limitada, no todo, algunas cosas, ampliar quizá un poco más las prescripciones que hacemos actualmente, pero no mucho más, o sea, tiene que ser con limitaciones" (E5).

"Entonces, es una responsabilidad súper potente, pero que va a requerir también de formarnos, 'deformarnos' algunas que ya estamos egresadas y que las mallas también vayan incorporando estos nuevos principios, o sea, aquí ya no te vas a poder quedar con la farmacología básica” (E7).

La subcategoría aplicación en área de desempeño surge en relación con cómo las enfermeras de atención primaria visualizan la prescripción enfermera aplicada exclusivamente en el área de ejercicio profesional donde se desempeñan, ya sea en atención infantil, de adultos, cuidados paliativos u otra.

"Soy enfermera de adulto... veo mayoritariamente pacientes con patológicas crónicas y.... entonces ahí nosotros como enfermeros podríamos ... tenemos el conocimiento para hacer la intervención necesaria para el bienestar del paciente" (E1).

"Yo lo encuentro genial, atingente a lo que tú vas a ver en tu atención, ejemplo, "¿porque no podría yo indicar las gotitas de fierro?... ¿por qué no? si yo a lo largo de todo el ciclo vital veo a ese niño desde los dos meses a los nueve años” (E3). 
En la dimensión simbólica del cuidar a través de la prescripción enfermera, se identificó la categoría formación profesional del enfermero. Ésta hace referencia a cómo las enfermeras consideran que la formación para ejecutar la prescripción es una construcción determinada por la interacción entre la formación teórica de pregrado y la farmacología aplicada en el ejercicio profesional. A partir de ésta surgen las subcategorías formación farmacológica de grado (de la enfermera) y experiencia.
La formación farmacológica de pregrado hace referencia al hecho de considerar que la formación teórica en farmacología recibida durante el período formativo profesional es insuficiente para asumir la prescripción, que es diferente a la actual administración de fármacos y educación asociada; pues esta situación estaría generando en las enfermeras una sensación de incertidumbre para la prescripción, la cual se vincula directamente con la subcategoría temor del componente afectivo del cuidar a través de la prescripción enfermera.

"Debería tener algún curso extra, para mí la formación de pregrado no basta, porque todos tenemos medio semestre o un año de farmacología ..., pero es poco” (E1).

"En el fondo vas a hablar de un fármaco, de una dosis, entonces también tiene que venir de la mano con una formación para nosotros mucha más potente en lo que es farmacología, o sea, ya no nos vamos a poder quedar con el semestre de la universidad que fue súper somero con respecto a eso y yo creo que también va a involucrar [a] las que ya estamos egresadas, un trabajo en cuanto a lo que es farmacología bastante más potente, o sea, con qué puedo, con qué no puedo, con qué interacciona” (E7).

La forma en que las enfermeras de atención primaria establecen que la aplicación de la enfermería contribuye a la construcción del profesional del área da lugar a la subcategoría experiencia. Se reconoce que la enfermera necesita de la interacción con otros profesionales y con los pacientes para aplicar los cuidados en forma de prescripción. Esta interacción, entendida como experiencia, es la que permite desenvolverse con mayor seguridad. En este sentido, se asume que a mayor cantidad de años laborales es mayor la seguridad para prescribir y, por tanto, también implica menos temor al prescribir.

“... yo ya sali de la universidad hace casi 5, 6 años y yo lo puedo ver ahora como en retrospectiva, cuando salí de la U puedo decir que no me sentía capaz de diagnosticar, o sea, de indicar un medicamento, pero uno con el tiempo, yo ya llevo casi 6 años trabajando aquí mismo, la expertiz más los estudios y la continuidad de seguirse especializando, yo creo que se puede dar...” (E1).

"Yo hace veinte años que estoy trabajando en el adulto y antes trabajaba en el programa de infantil..., uno conoce los efectos adversos de cada medicamento, tú sabes que si tie- 
ne una enfermedad renal no le puedes dar tal o cual cosa, ..., o si tiene una cardiopatía coronaria hay medicamentos que te afectan la parte cardiaca, uno sabe toda esas cosas, entonces nosotros perfectamente podríamos prescribir en todo lo que es programa, por ejemplo, como es programa de crónicos, programa de las heridas avanzada, curación avanzada..." (E4).

La subcategoría experiencia se encuentra en estrecha relación con la subcategoría actividad implícita, dado que las enfermeras de atención primaria establecen que la prescripción enfermera es una actividad que no puede ser realizada por enfermeras; sin embargo, en la práctica diaria la prescripción existe, o con fármacos de baja complejidad, o con un amplio margen terapéutico, o que se encuentra normado a nivel ministerial.

"La enfermera está limitada a dar solamente vitamina, fierro y tratamiento pediculosis, nada más, y podemos dar pasta lassar para la dermatitis micótica o la dermatitis del pañal, ni siquiera puedo dar clotrimazol para la dermatitis micótica, o sea, en el fondo serían cuatro medicamentos, vitaminas $C$ y D, fierro, launol que es la decametrina y la pasta lassar para la dermatitis del pañal” (E5).

“... en lo infantil, tenemos la autorización de prescribir ciertos medicamentos, como autorización interna para evitar sobrecarga de consultas médicas, y que son obvias y que de repente nosotros nos manejamos mejor, o por el tiempo que llevamos en los programas, que el propio médico que viene recién ingresando, entonces está incorporada la prescripción de algunos fármacos” (E6).

En la dimensión afectiva o actitud del cuidar a través de la prescripción enfermera, emergió la categoría valor atribuido, la cual surge en relación con la apreciación y el sentir que expresan las enfermeras con respecto al cuidado de enfermería manifestado como prescripción. En ella se identifican las subcategorías aporte positivo, in-

\section{diferencia y temor.}

La subcategoría aporte positivo da cuenta de las características positivas atribuidas a la prescripción, tanto desde la visión del desarrollo profesional, como desde la valoración que otorgarían los pacientes a la profesión enfermera.

“... [estaríamos] contribuyendo a derrocar un poco este estigma biomédico de dependencia del médico, yo creo que hemos avanzado bastante, enfermería ha avanzado bastante a sacarse esa dependencia (...) del médico y uno hacía simplemente, yo creo que contribuiría bastante, sería como la integralidad total, de un profesional de enfermería" (E1). 
“... el profesional de enfermería es el más integral dentro del equipo, porque vas a tener más atribuciones que antes (...), entonces la gente igual te va a mirar con otros ojos" (E2).

"Eso tiene que ver con la autonomía en la profesión, o sea, nosotros somos capaces de tomar decisiones y si somos capaces de tomar decisiones, obviamente somos capaces de prescribir, de gestionar el cuidado" (E4).

La subcategoría indiferencia recoge la valoración que algunas hacen de la prescripción enfermera, que si bien no constituye un aporte a la profesión tampoco sería algo negativo, y desde esta perspectiva es considerada un beneficio para el paciente, lo que establece una relación directa entre esta subcategoría y la de resolutividad asociada a la categoría beneficios sociales del componente figurativo del cuidar a través de la prescripción enfermera.

"Bajo mi criterio, siento que nosotros en nuestro actuar de enfermería como labor no dependemos de los fármacos, ¿ya?, siento que tú puedes hacer tu plan de cuidados, tu planificación con esa guagua o con ese adulto mayor, sin los medicamentos” (E3).

"Es súper bueno ganar una instancia como la de la prescripción, pero yo creo que tenemos que potenciar mucho la fármaco educación dirigida al paciente y familia, más que la prescripción” (E7).

La subcategoría temor surge en relación con cómo las enfermeras de atención primaria consideran que la prescripción enfermera, si bien es una actividad ejecutable, implica también asumir un riesgo en la atención del paciente. Esta sensación de temor estaría relacionada estrechamen- te con la falta de formación farmacológica de pregrado visualizada en la categoría formación profesional del enfermero en el componente simbólico del cuidar a través de la prescripción enfermera.

"A largo plazo los casos de negligencia [pueden aumentar], aumentan los casos de intoxicación medicamentosa, de interacciones medicamentosas y se hace sumario y se da cuenta de que son enfermeros la mayor cantidad" (E1).

"A mí me encantaría prescribir más, pero el riesgo que corres versus lo que ganas [es alto]" (E3).

La subcategoría temor se encuentra en estrecha relación con la subcategoría responsabilidad, pues asumir una nueva responsabilidad legal, en el componente afectivo del cuidar, es el principal agente desencadenante del sentimiento de temor en los profesionales de enfermería, el cual a su vez genera las limitaciones a la prescripción visualizadas en el componente figurativo del cuidar a través de la prescripción enfermera. 
“Te entrega una responsabilidad que tú antes no tenías, porque tú te dedicabas a educar sobre un fármaco que tú no entregabas, ahora no, ahora tú vas a tener que entregar el fármaco y, además, educar sobre éste, entonces por eso te digo tiene un pro y un contra legalmente claro" (E2).

“Claro, vas a tener que tener mucho más cuidado porque tú también vas a tener la facultad de entregar medicamentos. Tendría que ser con una capacitación previa porque no nos conviene hacernos responsables de cosas que no podemos manejar, tenemos que ser bien responsables en ese sentido" (E6).

En la Figura 1, se representa una síntesis de los componentes figurativo, simbólico y afectivo del cuidar a través de la prescripción enfermera, expuestos más arriba.

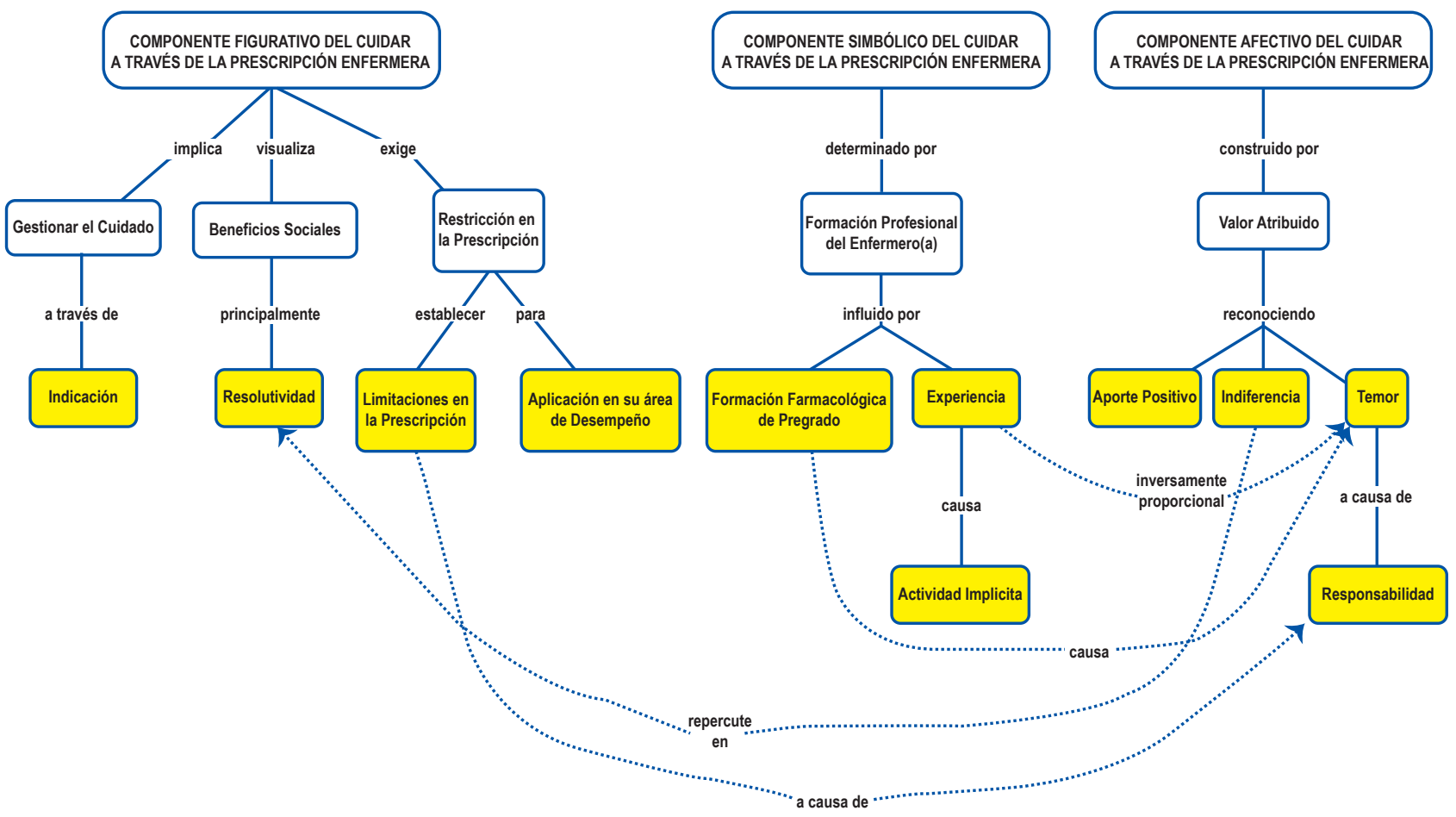

Figura 1. Representación social del cuidar a través de la prescripción enfermera.

Fuente: Elaboración propia.

\section{DISCUSIÓN}

Considerando que la teoría de las representaciones sociales aplicada en la investigación de enfermería basada en evidencia favorece la comprensión de la cosmovisión de las personas sobre el proceso salud-enfermedad-atención, haciendo posible el diseño y puesta en ejecución de planes terapéuticos pertinentes ${ }^{24}$, en este estudio se describen los componentes figurativo, simbólico y afectivo del cuidar a través de la prescripción enfermera. 
En relación al objetivo de describir el componente figurativo del cuidar mediante la prescripción enfermera que tienen las profesionales de enfermería de la red de atención primaria de salud de Temuco (Chile), se observa que la prescripción enfermera es vista como una herramienta para gestionar los cuidados a través de una indicación de enfermería, lo cual es similar al concepto adoptado por el Collegi Oficial d' Infermeria de Barcelona ${ }^{10}$. Asimismo, ella es considerada una herramienta de resolutividad que permite brindar una atención más expedita ${ }^{3}$, evitando la derivación hacia el profesional médico, lo cual coincide con el estudio realizado por de Fatima Fernandes, de Moura Lopes y Chaves Rocha, quienes demuestran que las enfermeras otorgan gran valor a la prescripción debido a que el médico no siempre está a disposición de la comunidad ${ }^{12}$.

Con respecto al componente figurativo de la representación social, las enfermeras consideran que la prescripción de fármacos es una actividad que debe estar legalmente regulada, centrada en un grupo farmacológico específico, asociado al área de desempeño laboral del profesional.

En relación al objetivo de describir el componente simbólico del cuidar mediante de la prescripción enfermera, se confirma que la formación en farmacología y los años de experiencia profesional son los principales factores que inciden en la información que se posee sobre la prescripción enfermera. Acerca de la formación farmacológica destaca la consideración de que ella es actualmente insuficiente para lograr asumir la facultad de forma eficiente ${ }^{25}$, lo que coincide con lo señalado por el Consejo General de Enfermería ${ }^{26}$ que indica que en los países donde se ha implementado la prescripción, las enfermeras han tenido que reformar sus conocimientos farmacológicos con diferentes programas de estudio para ser habilitadas en esta nueva función.

Acerca de la experiencia profesional, las enfermeras coinciden en que aquella adquirida durante el desempeño en APS otorga mayor seguridad para llevar a cabo la prescripción, tal como lo describen de Fátima Fernandes, de Moura Lopez y Chaves Rocha ${ }^{12}$, cuando plantean que la base académica de pregrado de los enfermeros no es la que les otorga seguridad en la prescripción, sino que son sus años de experiencia.

En cuanto al objetivo de describir el componente afectivo del cuidar mediante de la prescripción enfermera, las enfermeras valoran la prescripción como una actividad positiva que contribuye al desarrollo profesional, tal como señala un estudio que refiere que ella permite a las enfermeras proveer una asistencia más holística a los pacientes y que ayuda a integrar el curar y el cuidar $\frac{10}{10}$, así como el logro del desarrollo de la autonomía profesional ${ }^{27}, \underline{28}$. Sin embargo, hay también voces disidentes que consideran que más que ser un beneficio para la enfermería, la prescripción favorece exclusivamente al paciente ${ }^{29}$, lo cual coincide con un estudio de satisfacción usuaria que concluye que los pacientes están satisfechos y felices con la rapidez de la atención gracias a la prescripción enfermera, dado que disminuye el tiempo que se debe esperar para recibir una atención $\underline{28,30}$. 
En este componente también es interesante analizar el sentimiento de temor compartido por las enfermeras, el cual se relaciona directamente con el acto de asumir una nueva función dentro de sus prácticas y la responsabilidad que implica, lo cual representa un hallazgo no evidenciado en otros estudios, dado que en los países donde se ha implementado esta prescripción, los estrictos estándares en la formación farmacológica neutralizarían el temor y la inseguridad que la débil formación farmacológica de pregrado genera en las enfermeras.

Con todo lo anterior, se reconoce que una de las limitaciones de esta investigación, si no la principal, es el hecho de que la totalidad de participantes son de género femenino, lo cual, si bien da cuenta de una muestra homogénea, no permite discriminar en torno a la incidencia que eventualmente pudiese tener el género en la construcción de las representaciones sociales de la prescripción enfermera, pues no hay posibilidad de comparación con las representaciones generadas por profesionales de enfermería varones.

\section{CONCLUSIONES}

La presente investigación se planteó como objetivo describir los componentes figurativos (campo de representación), simbólico (información) y afectivo (actitudinal) del cuidar mediante la prescripción enfermera, que tienen los profesionales de enfermería de la red de atención primaria de salud. Al respecto, se puede sostener que ésta se encuentra compuesta por diferentes elementos que se conjugan entre sí para elaborarla, destacando que la prescripción enfermera es entendida como la acción de llevar a cabo una indicación de enfermería a fin de entregar un cuidado para la atención del paciente.

Para las participantes del estudio, asumir la prescripción debe ir acompañada de un marco regulatorio que las resguarde ética y legalmente, definiendo claramente los límites de esta facultad, principalmente por la responsabilidad legal que esta actividad implica. De esta forma, les otorgaría una mayor seguridad para realizar la prescripción y eliminaría el sentimiento de temor que se expresa en los discursos de las entrevistadas. Este grado de seguridad se encuentra influido por la insuficiente formación farmacológica de pregrado y los años de experiencia laboral, ante lo cual se considera que mientras menos años de experiencia profesional se tiene menor es el sentimiento de seguridad para prescribir; por el contrario, a mayor experiencia profesional, mayor es la seguridad. Asimismo, la prescripción sería una actividad idónea para la profesional de APS.

Se observan dos perspectivas, claramente definidas, sobre cómo los profesionales de APS definen la prescripción enfermera. Por un lado, están quienes la consideran como un elemento que contribuye al desarrollo y autonomía profesional en la toma de decisiones y, por otra parte, se encuentran quienes sostienen que la prescripción es sólo un aporte para el paciente. Sin embargo, ambas visiones coinciden en que es positiva para la profesión.

Es interesante analizar, además, en base a los antecedentes expuestos y sustentándose en la funciones orientadoras y justificadoras que poseen las representaciones sociales, que la postura y el 
comportamiento que tendrán las enfermeras de atención primaria, frente a la prescripción enfermera, en general es de aceptación de la función, siempre que se encuentre claramente definida en sus aspectos legales. No se observa ni manifiesta rechazo hacia esta prescripción.

\section{Conflicto de intereses: Los autores declaran no}

tener conflicto de intereses.

\section{REFERENCIAS}

1. Redondo L. Prescripción enfermera: Opinión de las enfermeras ante el nuevo Real Decreto 954/2015; 2016. Disponible en: http://uvadoc.uva.es/handle/10324/24512

2. Gómez RM, Afonso C, Hernández S, Gómez M. Actualidad en prescripción enfermera dentro de un equipo de salud. Revista ENE de Enfermería. 2011; 5(1): 5-24.

3. Gielen SC, Dekker J, Fracke AL, Mistiaen P, Kroezen M. The effects of nurse prescribing: A systematic review. Int J Nurs Stud. 2014; 51(7): 1048-61.

http://doi:10.1016/j.ijnurstu.2013.12.003

4. Romero-Collado A, Homs-Romero E, Zabaleta-delOlmo E, Juvinya-Canal D. Nurse prescribing in primary care in Spain: legal framework, historical characteristics and relationship to perceived professional identity. J Nurs Manag. 2014; 22(3): 394-404. http://doi:10.1111/jonm.12139

5. Reis Bellaguarda ML, Nelson S, Itayra Padilha M, Caravaca - Morera J. Prescriptive Authority and Nursing: a comparative analysis of Brazil and Canada. Rev Latino-Am Enfermagem. 2015; 23(6):1065-73. http://dx.doi.org/10.1590/0104-1169.0418.2650

6. Martiniano CS, Andrade PS, Magalhães FC, Souza FF, Clementino F, Uchôa SA. Legalização da prescrição de medicamentos pelo enfermeiro no Brasil: história, tendências e desafios. Texto \& Contexto - Enfermagem. 2015; 24(3): 809-17.

http://dx.doi.org/10.1590/0104-07072015001720014

7. Rodríguez S, Fuente N, Casado MI, Ayuso M. La prescripción enfermera en el Sistema Nacional de Salud: ¿realidad o ficción? Enfermería Clinica. 2015; 25(3):107-9. http://dx.doi.org/10.1016/j.enfcli.2015.05.001

8. Faeda M, Perroca M. Care management: agreement between nursing prescriptions and patients' care needs. Rev Latino-Am Enfermagem. 2016; 24: e2723. http://dx.doi.org/10.1590/1518-8345.0645.2723

9. Romero-Collado A, Raurell-Torreda M, Zabaleta-del-Olmo E, Rascon-Hernan C, Homs-Romero E. Nurse prescribing in Spain: The law and the curriculum. Nurs Health Sci. 2017; 19(3): 373-80.

http://doi:10.1111/nhs.12355

10. Contreras V, Martínez JI. Prescripción de enfermería en Chile. Horiz Enferm. 2012; 23(3):32-9.

http://doi:10.7764/Horiz Enferm.23.3.32

11. Cámara de Diputados. Sesión 27a. Legislatura $360^{\mathrm{a}}$; 2012; Valparaíso. p.127-130. Disponible en: http://www.camara.cl/pdf.aspx?prmID=9363\%20 \&prmTIPO $=$ TEXTOSESION

12. Fernandes MN, Lopes KS, Rocha FA. A prescrição de medicamentos na estratégia de saúde da família: percepção de enfermeiros. Rev Enferm UFSM. 2016; 6(3): 38292. http://doi10.5902/2179769220713

13. Moscovici S. El psicoanálisis, su imagen y su público. $1^{\mathrm{a}}$. ed. Buenos Aires: Editorial Huemul S.A; 1979.

14. Moscovici S. Psicología social. $1^{\mathrm{a}}$. ed. Barcelona: Ediciones Paidós; 1985.

15. Abric JC. Filosofía y cultura contemporánea. $1^{\mathrm{a}}$. ed. Colonia del Carmen: Ediciones Coyoacán; 2001.

16. Vivar C, Arantzamendi M, López-Dicastillo O, Gordo C. La teoría fundamentada como metodología de investigación cualitativa en enfermería. Index Enferm. 2010; 19(4): 283-8. https://doi.org/10.4321/S1132-12962010000300011

17. Paramo D. La teoría fundamentada (grounded theory), metodología cualitativa de investigación científica. Pensamiento \& Gestión. 2015;(39):119-46.

18. Hernández R, Fernández C, Baptista P. Metodología de la investigación. $6^{\mathrm{a}}$. ed. México D.F: McGraw-Hill Interamericana; 2014.

19. Arias Valencia MM, Giraldo Mora CV. El rigor científico en la investigación cualitativa. Investigación y Educación en Enfermería. 2011; 29(3): 500-14.

20. Lolas Stepke F, Quezada Sepúlveda A. Pautas éticas de investigación en sujetos humanos: nuevas perspectivas. Chile: Programa Regional de Bioética OPS/OMS; 2003. Disponible en: http://www.libros.uchile.cl/258

21. Consejo de Organizaciones Internacionales de las Ciencias Médicas y Organización Mundial de la Salud. Pautas éticas internacionales para la investigación biomédica enseres humanos; 2002. Disponible en: http://cioms. ch/wp-content/uploads/2016/08/PAUTAS ETICAS INTERNACIONALES.pdf

22. Asociación Médica Mundial. Declaración de Helsinki de la AMM - Principios éticos para las investigaciones médicas en seres humanos. 2015. Disponible en: http://www. wma.net/es/policies-post/declaracion-de-helsinki-de-laamm-principios-eticos-para-las-investigaciones-medicasen-seres-humanos/

23. Comisión Nacional para la Protección de los Sujetos Humanos de Investigación Biomédica y del Comportamiento. El informe Belmont. Principios y guías éticos para la protección de los sujetos humanos de investigación. 1979. Disponible en: http://www.bioeticayderecho. ub.edu/archivos/norm/InformeBelmont.pdf 
24. Ferreira M. Theory of social representations and contributions to the research of health care and nursing. ESC Anna Nery. 2016; 20(2): 216-17. http://dx.doi.org/10.5935/1414-8145.20160028

25. Scrafton J, McKinnon J, Kane R. Exploring nurses' experiences of prescribing in secondary care: informing future education and practice. J Clin Nurs. 2012; 21(13-14): 2044-53. http://doi:10.1111/j.1365-2702.2011.04050.x

26. Consejo General de Enfermería. Marco referencial para la prescripción enfermera. Documento base. 2015. Disponible en:

http://www.index-f.com/lascasas/documentos/lc0151.pdf

27. Cousins R, Donnell C. Nurse prescribing in general practice: a qualitative study of job satisfaction and work-related stress. Fam Pract. 2012; 29(2): 223-7. http://doi:10.1093/fampra/cmr077

28. Martín A. Enfermeria. Prescripción de fármacos y productos sanitarios. RECIEN Revista Científica de Enfermería. 2011; 1(3).

29. Teijlingen E, Hundley V. The importance of pilot studies. Nursing Standard. 2002;16(40): 33-6. http://doi:10.7748/ns2002.06.16.40.33.c3214

30. Armstrong A. Staff and patient views on nurse prescribing in the urgent-care setting. Nurse Prescribing. 2015;13(12): 614-19. http://doi.org/10.12968/npre.2015.13.12.614 